\title{
Investigation of Off-stoichiometry in Ternary Nitrides by EDS and HRTEM
}

\author{
Allison Mis ${ }^{1}$, Ann Greenaway ${ }^{2}$, Celeste Melamed ${ }^{1}$, Adele Tamboli ${ }^{2}$ and Geoff Brennecka ${ }^{1}$ \\ ${ }^{1}$ Colorado School of Mines, Golden, Colorado, United States, ${ }^{2}$ National Renewable Energy Laboratory, \\ Golden, Colorado, United States
}

Similarly to how III-Vs opened a new frontier for semiconductors, ternary nitrides are a promising family of semiconducting materials with a vast range of physical and optoelectronic properties. Ternary nitrides offer multiple avenues of band gap and lattice tunability, including cation ordering and off-stoichiometry. Multiple reports [1-2] illustrate that off-stoichiometry can have drastic effects on optical and electronic properties, in addition to the potential for formation of secondary phases. However, for many of these materials it is unclear whether off-stoichiometry is accommodated within the crystalline lattice via substitutional or interstitial defects, by clustering at grain boundaries, or through small amounts of secondary phases dispersed through the material. Off-stoichiometry poses a fundamental question that can have far-reaching consequences for measured properties and potential applications.

This study is complementary to combinatorial high-throughput materials discovery efforts in which structural and optoelectronic properties are studied as a function of cation composition. One of the more well-studied ternary nitrides, ZnGeN2, has recently been shown [3] to have properties which vary with cation off-stoichiometry and oxygen contamination. To better understand this phenomenon, offstoichiometric samples are examined using selected area electron diffraction (SAED), energy-dispersive spectroscopic (EDS) mapping, and high-resolution transmission electron microscopy (HRTEM).

These same techniques are also used to investigate $\mathrm{Zn}_{2} \mathrm{SbN}_{3}$, a novel ternary nitride which has shown promise as an optoelectronic semiconductor. [4] Highly off-stoichiometric $\mathrm{Zn}_{2} \mathrm{SbN}_{3}$, while still crystalline by x-ray diffraction, has an anomalous optical response. As shown in Fig. 1a, a cross-sectional bright field image of a thin-film specimen shows columnar grain growth at stoichiometric compositions (left), while at 46 cation\% $\mathrm{Sb}$, an amorphous layer sits atop the crystalline layer (right); this amorphous layer is assumed to be the cause of the anomalous optical properties. EDS mapping (Fig. 1b) shows that the amorphous layer is oxygen-rich and zinc-poor, with some zinc-containing intrusions from the layer below. This secondary layer was included in the sampling volume of high-throughput composition measurements, so previous assessments of the composition ranges for which this novel material forms must be examined and re-evaluated.

The growth of materials predicted computationally can often lead to more questions than answers, as in the case of $\mathrm{MgSnN} 2$, a material only recently realized in thin-film form. Fig. 2 shows $\mathrm{x}$-ray diffraction (XRD) patterns as a function of cation ratio in a sample of thin-film MgSnN2. [5] Previous work had assumed that MgSnN2 would take the ground-state wurtzite phase, peaks of which are visible throughout the sample. However, above 55 cation $\% \mathrm{Mg}$, a secondary rocksalt phase appears. This phase is metastable and was not predicted by computational efforts; its formation mechanism and distribution within the wurtzite material is studied using similar methods to those listed above. As with the two compounds above, investigating off-stoichiometry in ternary nitrides not only enriches the basic science understanding of these promising materials, but can provide necessary insight that enables production of high-quality material. 

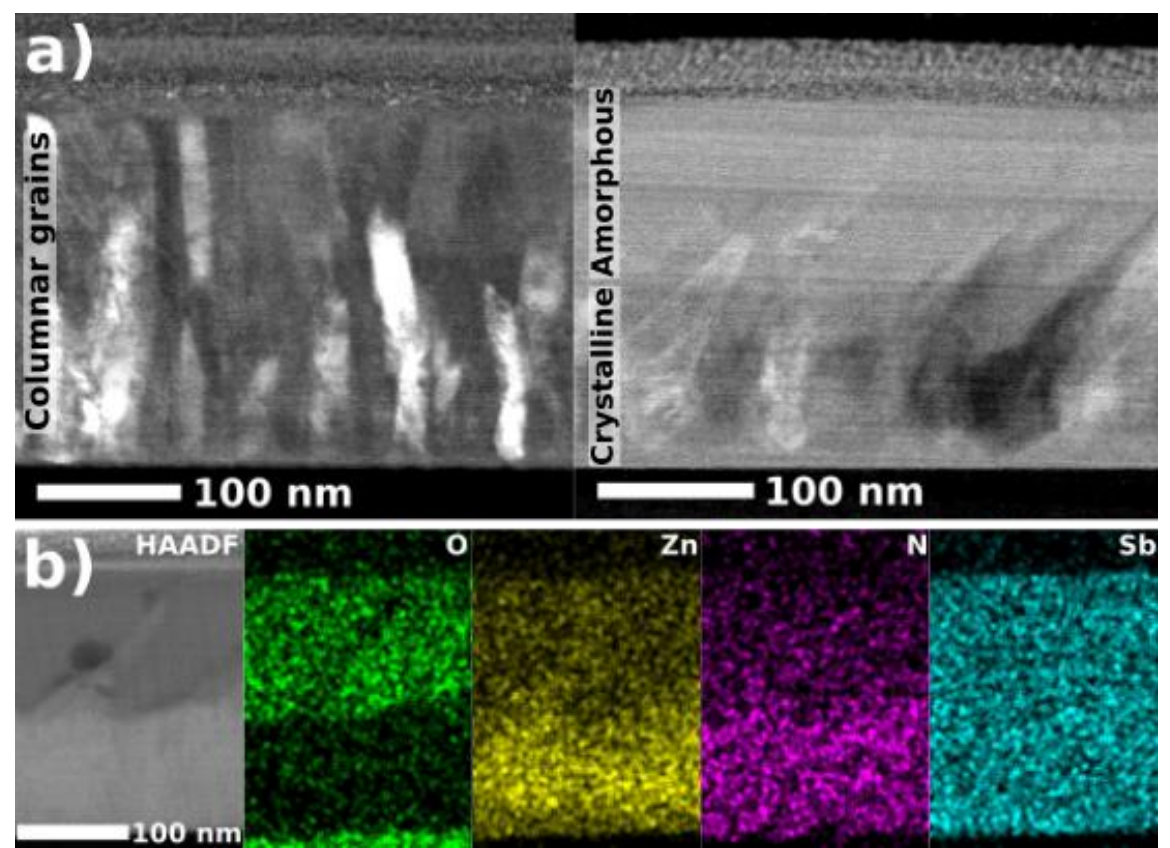

Figure 1. a) Bright field TEM of Zn2SbN3 illustrating columnar growth in a stoichiometric sample (left) and an amorphous layer in an Sb-rich sample (right).b) EDS mapping of an Sb-rich sample of Zn2SbN3 showing $\mathrm{O}, \mathrm{Zn}$, and $\mathrm{N}$ segregation.

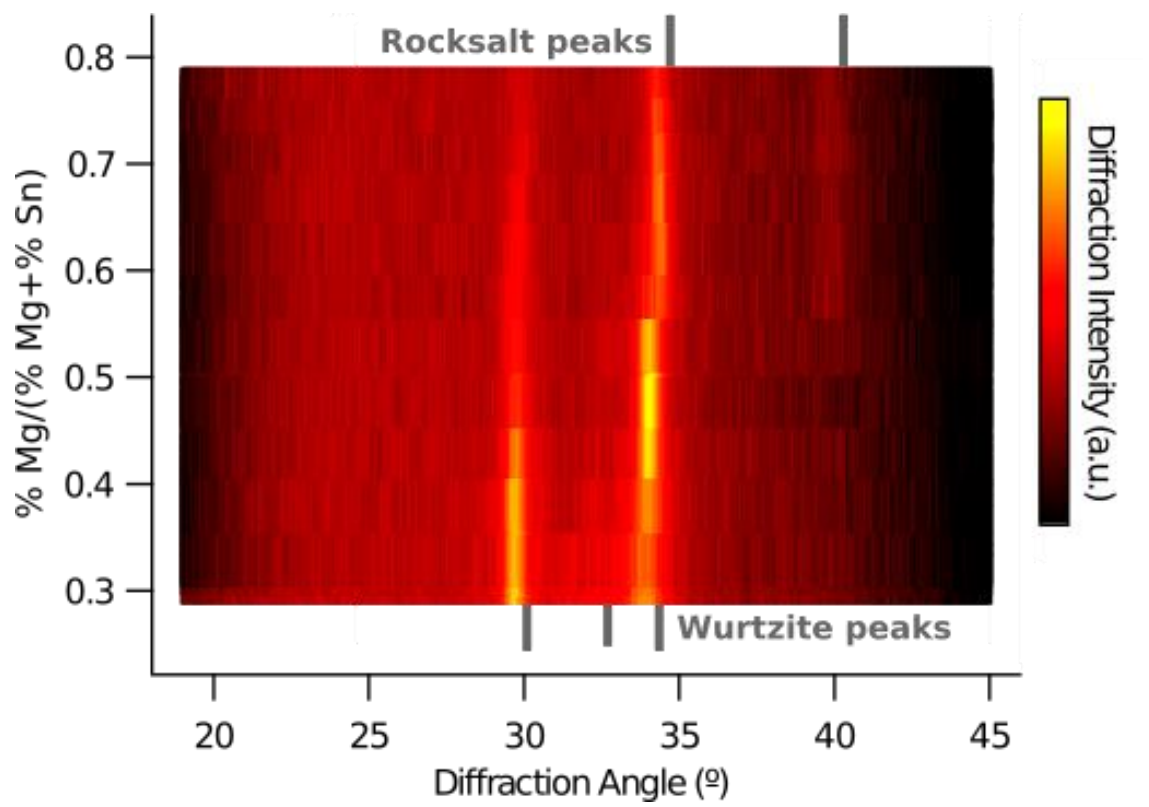

Figure 2. X-ray diffraction patterns as a function of cation composition for a sample of MgSnN2. The wurtzite phase exists throughout, with a coexisting rocksalt phase above 55 cation $\% \mathrm{Mg}$

References

1. Fioretti, et al., J. Mater. Chem 2015, 3, 11017-11028

2. Bauers et al., Jpn. J. Appl. Phys. 2019, 58 SC1015

3. Melamed, et al., In prep.

4. Arca, et al., Adv. Mat 2019,6, 1669-1674

5. Greenaway, et al. Submitted. 